\title{
The relationship between protein domains and homopeptides in the Plasmodium falciparum proteome
}

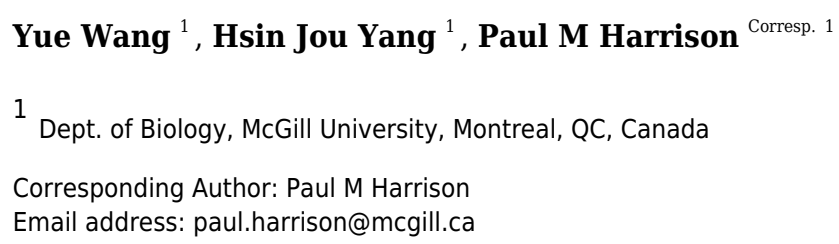

The proteome of the malaria parasite Plasmodium falciparum is notable for the pervasive occurrence of homopeptides or low-complexity regions (i.e., regions that are made from a small subset of amino-acid residue types). The most prevalent of these are made from residues encoded by adenine/thymidine (AT)-rich codons, in particular asparagine. We examined homopeptide occurrences within protein domains in P. falciparum. Homopeptide enrichments occur for hydrophobic (e.g., valine), or small residues (alanine or glycine) in short spans ( $<5$ residues), but these enrichments disappear for longer lengths. We observe that short asparagine homopeptides ( $<10$ residues long) have a dramatic relative depletion inside protein domains, indicating some selective constraint to keep them from forming. We surmise that this is possibly linked to co-translational protein folding, although there are specific protein domains that are enriched in longer asparagine homopeptides ( $\geq 10$ residues) indicating a functional linkage for specific poly-asparagine tracts. Top gene ontology functional category enrichments for homopeptides associated with diverse protein domains include 'vesicle-mediated transport', and 'DNA-directed 5'-3' RNA polymerase activity', with various categories linked to 'binding' evidencing significant homopeptide depletions. Also, in general homopeptides are substantially enriched in the parts of protein domains that are near/in IDRs. The implications of these findings are discussed. 
2 The relationship between protein domains and homopeptides

3 in the Plasmodium falciparum proteome

4

5 Short title: Domains and homopeptides in Plasmodium

6

7 Yue Wang ${ }^{1}$, Hsin-Jou Yang \& Paul M. Harrison ${ }^{1 *}$

8

$9{ }^{1}$ Department of Biology,

10 McGill University,

11 Montreal, QC,

12 Canada.

13

$14{ }^{*}$ Correspondence:

15 Paul M. Harrison

16 Email: paul.harrison@mcgill.ca

17

18 


\section{Abstract:}

20 The proteome of the malaria parasite Plasmodium falciparum is notable for the pervasive

21 occurrence of homopeptides or low-complexity regions (i.e., regions that are made from

22 a small subset of amino-acid residue types). The most prevalent of these are made from

23 residues encoded by adenine/thymidine (AT)-rich codons, in particular asparagine. We

24 examined homopeptide occurrences within protein domains in $P$. falciparum.

25 Homopeptide enrichments occur for hydrophobic (e.g., valine), or small residues (alanine

26 or glycine) in short spans ( $<5$ residues), but these enrichments disappear for longer

27 lengths. We observe that short asparagine homopeptides ( $<10$ residues long) have a

28 dramatic relative depletion inside protein domains, indicating some selective constraint to

29 keep them from forming. We surmise that this is possibly linked to co-translational protein

30 folding, although there are specific protein domains that are enriched in longer asparagine

31 homopeptides ( $\geq 10$ residues) indicating a functional linkage for specific poly-asparagine

32 tracts. Top gene ontology functional category enrichments for homopeptides associated

33 with diverse protein domains include 'vesicle-mediated transport', and 'DNA-directed 5'-

34 3' RNA polymerase activity', with various categories linked to 'binding' evidencing

35 significant homopeptide depletions. Also, in general homopeptides are substantially

36 enriched in the parts of protein domains that are near/in IDRs. The implications of these

37 findings are discussed.

39 Introduction

Plasmodium falciparum (Pf) is a single-celled protozoan that causes malaria in

41 humans. Malaria causes hundreds of thousands of deaths every year, with $\sim 405,000$ in 
422018 (Global Malaria Programme 2019). Treatment for malaria is confounded by its ability

43 to adapt quickly to drugs and to the human immune system; its antigenic diversity is a

44 major problem for vaccine development (Ferreira et al. 2004; Ferreira et al. 2003; Freitas-

45 Junior et al. 2000). The complete genome sequence of $P f$ contains $>5000$ protein-coding

46 genes (Gardner et al. 2002). Early analysis indicated that low-complexity regions (i.e.,

47 regions that consist mostly of a small subset of amino-acid types) or homopeptides (runs

48 of single amino acids) are a prominent feature of the encoded proteins, with more than

49 half of proteins being low-complexity over most of their sequences (Pizzi \& Frontali 2001).

50 Asparagine-rich regions are the most abundant (An \& Harrison 2016; Pizzi \& Frontali

51 2001). The low-complexity regions (LCRs) have been postulated to have a function

52 primarily at the nucleotide level (Xue \& Forsdyke 2003). Their abundance depends largely

53 on genomic $A+T$ or $G+C$ content (DePristo et al. 2006; Xue \& Forsdyke 2003), and they

54 also acquire further low-complexity insertions and deletions according to a power-law

55 rule: that is, longer LCRs acquire longer insertions/deletions (DePristo et al. 2006). Pf

56 LCRs can be classified into three distinct types, including a high $G+C$ type that is linked

57 to recombination hotspots (Zilversmit et al. 2010). There is a pattern of enrichment of long

58 intergenic poly(AT) tracts in Plasmodium species, some of which are immediately

59 adjacent to genes and run into them (Russell et al. 2014). Although asparagine is

60 preferred in LCRs of Pf, a different residue type with AT-rich codons (lysine) is more

61 prominent in the CVK group, which is a set of four primate-infecting plasmodia (Chaudhry

62 et al. 2018). As well as being sites of polymorphic variation themselves (Chaudhry et al.

63 2018), Pf LCRs are linked to increased single-nucleotide polymorphism in their vicinity

64 (Haerty \& Golding 2011). 
Although homopeptides are sites of such polymorphic variation, earlier work 66 showed that some homopeptides are deeply conserved across orthologs from bacteria

67 and eukaryotes, suggesting ancient origin and functional essentiality (Faux et al. 2005).

68 In general, homopeptides are more conserved in bacteria, than in archaea and eukaryotes, and there is a correlation between repeat length differences and species divergence (Uthayakumar et al. 2012). Homopeptides increase the functional versatility

71 of proteins, and facilitate spatial organization of proteins in a repeat-dependent way 72 (Chavali et al. 2017). They are also significantly linked to many human diseases (Lobanov 73 et al. 2016).

Low-complexity regions rich in hydrophilic residues are significantly associated with protein intrinsic disorder (Delucchi et al. 2020; Romero et al. 2001). Previous surveys have shown that $10-40 \%$ of $P f$ LCR residues are predicted as intrinsically disordered in tracts $\geq 40$ residues long, and that $>60 \%$ of sequences have such a tract (Feng et al. 2006; Mohan et al. 2008). Such annotated disordered regions in Pf are significantly depleted of predicted MHC-binding peptides, which has implications for vaccine development, since many vaccine target proteins are intrinsically disordered (Guy et al. 2015). Low-complexity regions rich in asparagine (and/or glutamine) are common in 82 domains that form prions (i.e., self-propagating amyloid particles) (Harbi \& Harrison 83 2014a; Harbi \& Harrison 2014b; Harbi et al. 2012; Harrison 2017; Su \& Harrison 2019).

84 In budding yeast (Saccharomyces cerevisiae), propagation of these particles can be 85 sustained during budding, mating and laboratory protocols (Harbi \& Harrison 2014c).

86 Predicted prions have been detected in all the domains of life (Espinosa Angarica et al. 87 2013), including thousands in viruses and phages (Tetz \& Tetz 2017; Tetz \& Tetz 2018), 
88 and tens of thousands in bacteria (Harrison 2019). Pf has prion-like domains (that arise

89 in asparagine-rich LCRs) in $10-24 \%$ of its proteins (Singh et al. 2004) (An \& Harrison

90 2016; Pallares et al. 2018). Just like Pf, there are Saccharomycetes fungi that have high

91 proportions of prion-like proteins with poly-asparagine in them (An et al. 2016). There is

92 some evidence that asparagine-rich LCRs act as 'tRNA sponges' that slow down the

93 translation rate of proteins to aid in co-translational folding (Filisetti et al. 2013; Frugier et

94 al. 2010). This is a type of parallel function at the DNA/RNA level for which there is

95 increasing evidence for intrinsically disordered regions in proteins (Pancsa \& Tompa 96 2016).

97 Here, we investigate the relationship between homopeptides and both defined 98 protein domains and intrinsic disorder in Pf proteins. We observe significant depletions in 99 homopeptides for specific types of amino acid, in particular asparagine and aspartate.

100 Homopeptides are substantially enriched in the parts of protein domains that are near or 101 in intrinsically disordered regions (IDRs).

102

103 Methods

104 Source Data

105 The UniProt (Boeckmann et al. 2003) reference proteome for Plasmodium 106 falciparum strain 3D7 was downloaded from www.uniprot.org in January 2019. Protein 107 domain annotations for $P f$ were taken from the Pfam database (El-Gebali et al. 2019). For 108 comparative analysis, three further reference proteomes were downloaded from the same 109 source for the following: another $P$. falciparum strain $(\mathrm{FCH} / 4)$, P. yoelii (strain 17XNL), 110 and $P$. vivax (strain Salvador I). 


\section{Annotation}

113 Homopeptides were defined as repetitions of one amino-acid type with a minimum

114 length of 3 residues (Figure 1).

115 Proteins were annotated for intrinsic disorder using the DISOPRED3 and

116 IUPRED2a programs (Dosztanyi et al. 2005; Huntley et al. 2015; Ward et al. 2004).

117 IUPRED2a operates on inputted single sequences, and predicts intrinsic disorder by

118 estimating inter-residue interaction energies (Erdos \& Dosztanyi 2020). It was the best

119 performing single-sequence method for intrinsic disorder annotation, with an AUC (area-

120 under-curve) value of 0.83 for the ROC curve in a recent assessment (Nielsen \& Mulder

121 2019); DISOPRED3 also had a value of $A U C=0.83$ in this assessment, and was one of

122 the best performing methods that use evolutionary information as input. Only regions of

123 predicted disorder $\geq 30$ residues long were considered. A 30-residue length cut-off was

124 used since this is a common threshold or boundary value used in characterizing

125 intrinsically-disordered regions, or in training algorithms for prediction of intrinsic disorder

126 (Atkins et al. 2015). Thus, we used the default 'long' parameter choice for the IUPRED2a

127 program. Also, the DISOPRED3 program was run with a $2 \%$ expected false positive rate

128 for the algorithm training set, which is in is the author's recommended parameter range

129 (Jones \& Cozzetto 2015). The results from either intrinsic disorder annotation program

130 were considered separately.

131

\section{Enrichments \& depletions}


134 disorder on the occurrence of homopeptides in $P f$, we checked whether there is any

135 deviation from random placement for homopeptides within protein domains and

136 annotated intrinsic disorder. These can be either enrichments relative to background

137 populations or depletions. The background populations were either the whole proteome

138 or the set of protein domain annotations as described below. Enrichments and depletions

139 for homopeptides in protein domains were calculated as depicted (Figure 1). These were

140 determined for individual amino-acid types in homopeptides using equation (1) below for

141 hypergeometric probability, for sampling with replacement:

$$
P(k)=\frac{\left(\begin{array}{l}
K \\
k
\end{array}\right)\left(\begin{array}{l}
N-K \\
n-k
\end{array}\right)}{\left(\begin{array}{l}
N \\
n
\end{array}\right)}
$$

145 with the sample counts given by:

$$
k=\text { number of residues in homopeptides in domains of one amino-acid type }
$$$$
n=\text { number of residues in homopeptides in domains }
$$

and the background counts given by the quantities: $N=$ number of residues in homopeptides in the proteome

154 types were also calculated with the sample counts given by: 
155

158

161

162

163 164

172 Proximity to IDRs was determined using a 10-residue buffer at either end of the annotated

173 IDRs (Figure 1).

174

175

$$
\begin{aligned}
& k=\text { number of residues in homopeptides in one domain type } \\
& n=\text { number of residues in homopeptides in all domain types }
\end{aligned}
$$

and the background counts given by the quantities:

$$
K=\text { number of residues in one domain type }
$$

$$
N=\text { number of residues in all domain-types }
$$

Enrichments and depletions for protein domains overlapping or near annotated intrinsically disordered regions (IDRs) were also calculated with the sample counts given by:

and the background counts given by the quantities:

$$
\begin{aligned}
& K=\text { number of residues in one domain type } \\
& N=\text { number of residues in all domain-types }
\end{aligned}
$$

$k=$ number of residues in one domain type which near or inside disordered regions $n=$ number of residues in all domain types which near or inside disordered regions 
$178 \quad$ All enrichments/depletions were calculated using hypergeometric probability with

179 appropriate Bonferroni corrections for multiple hypothesis testing. For example, the

180 Bonferroni correction for enrichments/depletions of homopeptides of individual amino

181 acids in protein domains was $P=0.05 / 20=0.0025$, since there are 20 different amino

182 acids being sampled from the same background population.

183

184 Propensity

185 A propensity for homopeptides of a specific amino-acid type to occur in protein 186 domains $\left(\mathbf{P}_{\text {dom }}\right)$ was calculated as:

187

$$
\mathbf{P}_{\text {dom }}=\log _{10}[(\mathrm{k} / \mathrm{n}) /(\mathrm{K} / \mathrm{N})]
$$

188 The values of $\mathrm{k}, \mathrm{n}, \mathrm{K}$ and $\mathrm{N}$ are as listed above just below equation (1). This was 189 calculated for the homopeptide threshold $\geq 3$ residues.

\section{Results}

\section{Enrichments and depletions of homopeptides in protein domains} Homopeptides are abundant and pervasive in the Pf proteome, yet it is not clear

194 from a structural perspective which homopeptides are more tolerated in protein domains. We analysed the preferences of homopeptides of each specific amino-acid type for

196 insertion into protein domains, using three different length thresholds for homopeptides

197 (Table 1). The statistical enrichments/depletions are listed, as well as the fraction of the

198 homopeptide populations for each amino acid, and the propensity $\left(\mathbf{P}_{\text {dom }}\right)$ of homopeptides

199 of each amino acid for protein domains, calculated as described in Methods. For the 200 minimum homopeptide threshold of $\geq 3$ residues length, the most enriched amino acids 
201 include the major aliphatic hydrophobic residues valine, isoleucine and leucine, which is

202 to be expected because of the extensive hydrophobic cores of protein domains. Also, the

203 small residues alanine and glycine exhibit highly significant enrichments. For these

204 amino-acid types, the enrichments are only for short homopeptides (of size 3 or 4

205 residues), since the enrichments disappear for longer homopeptide thresholds (Table 1).

206 Positively-charged homopeptides and other hydrophilic homopeptides are also generally

207 enriched (lysine, arginine, serine, threonine), while negatively-charged homopeptides are

208 significantly depleted or show no preferences. Lysine homopeptides are the second most

209 abundant in the proteome and are made from AT-rich codons; their relationships with

210 specific protein domains are discussed below. Some amino acids show an enrichment,

211 with a comparable propensity for structural domains $\left(\mathbf{P}_{\text {dom }}\right)$ as for other amino acids, but

212 these are not significant. Most strikingly though, short asparagine homopeptides are

213 highly significantly depleted within protein domains.

214 Histograms of homopeptide length also indicate that within protein domains,

215 homopeptides generally lack the longer homopeptide lengths ( $\geq 10$ residues) that make

216 up the majority of homopeptides outside of protein domains (Figure 2).

217 For the longest homopeptide threshold ( $\geq 10$ residues length), the number of

218 amino-acid types which are comparatively tolerated in protein domains dramatically

219 decreases to one (asparagine; Table 1). Keeping in mind that the enrichment calculations

220 are effectively based on the comparison of different amino-acid types, there should

221 always be at least one enriched amino-acid type unless there are completely no

222 homopeptides at all at a certain threshold. Poly-asparagine homopeptides are depleted

223 in domains until the threshold is extended to 10 , which leaves it as the only one existing 
224 in domains. The enrichment observed for longer polyasparagine tracts ( $\geq 10$ residues,

225 Table 1) arises from a small number of specific protein domains that may have a

226 functional linkage for these polyasparagine tracts (Table S1), for example for specific

227 protein interactions.

The enrichment/depletion results for individual amino-acid types are little affected

229 by the boundary definition of protein domains (i.e., chopping off 3, 5 or 7 residues from

230 the ends of the domains, Table S3), with just some enrichments for glutamine and

231 glutamate becoming significant for these shortened domains. This indicates that these

232 homopeptides significantly occur near the ends of protein domains.

Since lysine and arginine homopeptides are in general significantly enriched in

234 protein domains, and asparagine and aspartate significantly depleted, we examined

235 which individual protein domains are linked to these trends (Table S1). Despite the

236 substantial general depletion of asparagine homopeptides within protein domains, there

237 are 87 individual domains with significant enrichment of asparagine homopeptides,

238 including the low-copy-number Sin-like region and the Sacl homology domain; these also

239 stand out when we restrict the analysis to polyasparagine tracts $\geq 10$ residues long (Table

240 S1). The most prominent lysine homopeptide enrichments are for Rifin and PfEMP DBL

241 domains. Specific domains are also linked to hydrophobic or small-residue homopeptides,

242 such as glycine homopeptides arising for ribosomal proteins (Table S1).

244 Gene Ontology enrichments 
We examined the enrichments and depletions of Gene Ontology functional

246 categories associated with homopeptides in protein domains (Table S2). Some top

247 enrichments of Gene Ontology (GO) functional categories for homopeptides in protein

248 domains include: GO:0003899 (DNA-directed 5'-3' RNA polymerase activity), an

249 enrichment caused by seven different protein domains, and GO:0042578 (phosphoric

250 ester hydrolase activity), which is unique to the Sacl homology domain (which is involved

251 in clathrin-mediated endocytosis; 3 copies in Pf) (Table S2). Inspection of other GO

252 category enrichments indicate that they are also caused by diverse protein domains, e.g.,

253 GO:0016192 (vesicle-mediated transport) which is linked to homopeptide enrichments in

25411 different protein domains, pointing to specific functional significance for homopeptides

255 in the interaction of these proteins. Nonetheless, in general 'protein binding' is significantly

256 depleted in the list (Table S2), as are the other high-level 'RNA-binding' and 'GTP-binding'

257 terms.

The relationship between specific protein domain homopeptides and intrinsic

disorder

IDRs tend to have homopeptides and low-complexity sequences in them (Romero

262 et al. 2001). We surmised that the relationship of different protein domains with

263 homopeptides might be caused by their proximity to or overlap with intrinsically disordered

264 regions (IDRs) of proteins. In general, homopeptides are enriched in the parts of protein

265 domains that are near or in IDRs (Table 2; results for either the DISOPRED3 or IUPred2A

266 program are shown). Also, there is only one individual protein domain type that is 
267 significantly depleted in homopeptides near/in IDRs, with the remainder of significant

268 deviations being enrichments (Table 2).

269

270 Comparison of trends in other Plasmodia

271 The trends observed for Pf strain 3D7 were validated by analysis of another Pf

272 strain $(\mathrm{FCH} / 4)$ that was picked from the UniProt reference proteome list (Boeckmann et

273 al. 2003) (Table S4). There is just one small change with enrichments of glutamate

274 homopeptides in protein domains becoming significant (Table S4). Comparisons were

275 also made with proteomes of $P$. yoelii, a malaria parasite of rodents, and $P$. vivax, a

276 member of the CVK group of primate-infecting plasmodia (Chaudhry et al. 2018). P. yoelii

277 has an overall approximately even predominance of $\mathrm{N}$ and $\mathrm{K}$ homopeptides, and $P$. vivax

278 has predominance of $\mathrm{K}$ homopeptides rather than of $\mathrm{N}$ homopeptides (Table S4). The

279 depletion of $\mathrm{N}$ homopeptides in protein domains is maintained in $P$. yoelii, but there is no

280 significant depletion/enrichment in P. vivax. K homopeptides also become significantly

281 depleted within protein domains in $P$. yoelii, despite their similar overall levels to $\operatorname{Pf}(29 \%$

282 in P.yoelii versus $23 \%$ in Pf). The results for homopeptide enrichments in parts of protein

283 domains overlapping IDRs (Table 2) also remain highly significant for these three other

284 Plasmodia proteomes (P-values $\sim 0.0)$.

285

286 Discussion

287 Homopeptide trends 
V) or small ( $A$ or $G$ ) residues, disappear at longer lengths ( $\geq 5$ residues). This indicates a

290 limit to their toleration within protein domain cores, for example because they are not so

291 easily accommodated in regular secondary structures.

We observed a substantial significant relative depletion of short asparagine runs

( $<10$ residues long) in protein domains. Plasmodia have acquired great amounts of $\mathrm{N}$

homopeptide tracts during evolution, but statistically these have not been appearing or

'landing' within domains. The lack of short intra-domain asparagine runs may be because they interfere with protein folding in some way. For example, they may slow down cotranslational protein folding due to a lack of asparaginyl-tRNAs, since levels of asparaginyl-tRNAs in Pf are normal despite the high amounts of asparagine in their coding sequences (Filisetti et al. 2013; Frugier et al. 2010). Thus asparagine homopeptides may be 'tRNA sponges' that soak up tRNAs and slow down translation and co-translational folding (Filisetti et al. 2013; Frugier et al. 2010). It is possible that homopeptides, and in particular poly-asparagine homopeptides may make protein 303 domains more prone to misfolding. Although generally slower translation is thought to aid 304 in correct co-translational folding (Waudby et al. 2019), sometimes faster translation is

305 more desirable through segments that are prone to misfolding (O'Brien et al. 2014), or for 306 translational efficiency at buried residue sites or sites that are vulnerable to structurally

307 disruptive mutations (Wang et al. 2015; Zhou et al. 2009). However, experiments with Pf 308 chaperone Hsp110c, have shown that $P f$ has cellular mechanisms that are designed to 309 prevent aggregation linked to asparagine tracts (Muralidharan \& Goldberg 2013). A few

310 specific protein domains have enrichment of long polyasparagine tracts. Such tracts may 
311 have a specific functional role in these proteins, perhaps for protein or nucleic-acid

312 interaction. Another possibility might be that correct folding of these specific domains is

313 not affected by slow rates of translation, thus $\mathrm{N}$ homopeptides can arise in them because

314 of their general abundance in the proteome. Interestingly, the significant depletion of $\mathrm{N}$

315 homopeptides is maintained in the rodent malaria pathogen $P$. yoelii, but there is an

316 absence of significant depletion/enrichment in the more distantly related $P$. vivax malaria

317 pathogen from the CVK group. This may indicate that the $\mathrm{N}$ homopeptides in the $P$. vivax

318 protein domains are reduced mainly to those that have functional roles.

\section{Gene Ontology enrichments}

In the gene ontology enrichments/depletion analysis we see a general trend for

322 depletion of functional categories associated with 'binding' (protein binding; RNA binding;

323 GTP binding; ion binding). This suggests that homopeptides may be selected against in

324 structured interaction interfaces, perhaps since they introduce a lack of interaction

325 specificity, or increase the likelihood of off-target binding. Also, the gene ontology results

326 indicate that homopeptide occurrences may be useful information for the discrimination

327 of protein function from the analysis of sequences (Huntley et al. 2014; Huntley et al.

328 2015; Jiang et al. 2016; Le et al. 2019a; Le et al. 2019b; Mutowo-Meullenet et al. 2013).

\section{Intrinsic disorder}

We surmise that homopeptides in protein domains are enriched in the parts of the

332 domains near or in IDRs because IDRs generally have more tolerance for 
333 insertions/deletions, and are the main determinants of changes in protein length over

334 evolution (Light et al. 2013). Also, our results indicate that the parts of protein domains

335 that can become intrinsically disordered are enriched in homopeptides relative to other

336 domain parts. This may be an important part of encrypting their ability to transition

337 structurally (Narasumani \& Harrison 2015).

\section{Conclusions}

The most pervasive homopeptide in Plasmodium falciparium, poly-asparagine, is

341 substantially depleted within protein domains, whereas other homopeptides that we might

342 expect in the hydrophobic core of domains, such as poly-leucine or -valine or -isoleucine,

343 and other generally abundant homopeptides (such as lysine) are enriched at the shorter

344 homopeptide lengths studied. We hypothesize that generally poly-asparagine formation

345 is repressed inside protein domains because its occurrence may slow co-translational

346 folding (Filisetti et al. 2013; Frugier et al. 2010), which might be problematic within a

347 protein domain that has only partially been translated. (Scenarios in which both transient

348 fast and slow folding may be problematic for co-translational protein folding are possible

349 (O'Brien et al. 2014)). Further experimental work is needed to investigate these

350 hypotheses. In general, homopeptides are depleted for functional categories associated

351 with diverse types of binding, indicating that they may interfere with specificity in

352 structured interfaces. However, the parts of protein domains that can become intrinsically

353 disordered have homopeptide enrichment relative to other parts of domains. Since some 354 domains fold upon binding to other proteins, and the parts of protein domains that overlap 
355

356

357

358

359

360

361

362

363

364

365

366

367

368

369

370

371

372

373

374

375

376

377

378

379

380

381

382

383

384

385

386

387

388

389

390

391

392

393

394

395

396

intrinsic disorder have such homopeptide enrichment, these results suggest that protein

homopeptides may be useful in effecting such structural transitions.

\section{References}

An L, Fitzpatrick D, and Harrison PM. 2016. Emergence and evolution of yeast prion and prionlike proteins. BMC Evol Biol 16:24. 10.1186/s12862-016-0594-3

An L, and Harrison PM. 2016. The evolutionary scope and neurological disease linkage of yeastprion-like proteins in humans. Biol Direct 11:32. 10.1186/s13062-016-0134-5

Atkins JD, Boateng SY, Sorensen T, and McGuffin LJ. 2015. Disorder Prediction Methods, Their Applicability to Different Protein Targets and Their Usefulness for Guiding Experimental Studies. Int J Mol Sci 16:19040-19054. 10.3390/ijms160819040

Boeckmann B, Bairoch A, Apweiler R, Blatter MC, Estreicher A, Gasteiger E, Martin MJ, Michoud K, O'Donovan C, Phan I, Pilbout S, and Schneider M. 2003. The SWISS-PROT protein knowledgebase and its supplement TrEMBL in 2003. Nucleic Acids Res 31:365370.

Chaudhry SR, Lwin N, Phelan D, Escalante AA, and Battistuzzi FU. 2018. Comparative analysis of low complexity regions in Plasmodia. Sci Rep 8:335. 10.1038/s41598-017-18695-y

Chavali S, Chavali PL, Chalancon G, de Groot NS, Gemayel R, Latysheva NS, Ing-Simmons E, Verstrepen KJ, Balaji S, and Babu MM. 2017. Constraints and consequences of the emergence of amino acid repeats in eukaryotic proteins. Nat Struct Mol Biol 24:765-777. $10.1038 / \mathrm{nsmb} .3441$

Delucchi M, Schaper E, Sachenkova O, Elofsson A, and Anisimova M. 2020. A New Census of Protein Tandem Repeats and Their Relationship with Intrinsic Disorder. Genes (Basel) 11. 10.3390/genes 11040407

DePristo MA, Zilversmit MM, and Hartl DL. 2006. On the abundance, amino acid composition, and evolutionary dynamics of low-complexity regions in proteins. Gene 378:19-30. 10.1016/j.gene.2006.03.023

Dosztanyi Z, Csizmok V, Tompa P, and Simon I. 2005. IUPred: web server for the prediction of intrinsically unstructured regions of proteins based on estimated energy content. Bioinformatics 21:3433-3434. 10.1093/bioinformatics/bti541

El-Gebali S, Mistry J, Bateman A, Eddy SR, Luciani A, Potter SC, Qureshi M, Richardson LJ, Salazar GA, Smart A, Sonnhammer ELL, Hirsh L, Paladin L, Piovesan D, Tosatto SCE, and Finn RD. 2019. The Pfam protein families database in 2019. Nucleic Acids Res 47:D427-D432. 10.1093/nar/gky995

Erdos G, and Dosztanyi Z. 2020. Analyzing Protein Disorder with IUPred2A. Curr Protoc Bioinformatics 70:e99. 10.1002/cpbi.99

Espinosa Angarica V, Ventura S, and Sancho J. 2013. Discovering putative prion sequences in complete proteomes using probabilistic representations of $\mathrm{Q} / \mathrm{N}$-rich domains. $B M C$ Genomics 14:316. 10.1186/1471-2164-14-316

Faux NG, Bottomley SP, Lesk AM, Irving JA, Morrison JR, de la Banda MG, and Whisstock JC. 2005. Functional insights from the distribution and role of homopeptide repeat-containing proteins. Genome Res 15:537-551. 10.1101/gr.3096505 
397

398

399

400

401

402

403

404

405

406

407

408

409

410

411

412

413

414

415

416

417

418

419

420

421

422

423

424

425

426

427

428

429

430

431

432

433

434

435

436

437

438

439

440

441
Feng ZP, Zhang X, Han P, Arora N, Anders RF, and Norton RS. 2006. Abundance of intrinsically unstructured proteins in P. falciparum and other apicomplexan parasite proteomes. Mol Biochem Parasitol 150:256-267. 10.1016/j.molbiopara.2006.08.011

Ferreira MU, da Silva Nunes M, and Wunderlich G. 2004. Antigenic diversity and immune evasion by malaria parasites. Clin Diagn Lab Immunol 11:987-995. 10.1128/CDLI.11.6.987-995.2004

Ferreira MU, Ribeiro WL, Tonon AP, Kawamoto F, and Rich SM. 2003. Sequence diversity and evolution of the malaria vaccine candidate merozoite surface protein-1 (MSP-1) of Plasmodium falciparum. Gene 304:65-75. 10.1016/s0378-1119(02)01180-0

Filisetti D, Theobald-Dietrich A, Mahmoudi N, Rudinger-Thirion J, Candolfi E, and Frugier M. 2013. Aminoacylation of Plasmodium falciparum tRNA(Asn) and insights in the synthesis of asparagine repeats. J Biol Chem 288:36361-36371. 10.1074/jbc.M113.522896

Freitas-Junior LH, Bottius E, Pirrit LA, Deitsch KW, Scheidig C, Guinet F, Nehrbass U, Wellems TE, and Scherf A. 2000. Frequent ectopic recombination of virulence factor genes in telomeric chromosome clusters of P. falciparum. Nature 407:1018-1022. $10.1038 / 35039531$

Frugier M, Bour T, Ayach M, Santos MA, Rudinger-Thirion J, Theobald-Dietrich A, and Pizzi E. 2010. Low Complexity Regions behave as tRNA sponges to help co-translational folding of plasmodial proteins. FEBS Lett 584:448-454. 10.1016/j.febslet.2009.11.004

Gardner MJ, Hall N, Fung E, White O, Berriman M, Hyman RW, Carlton JM, Pain A, Nelson KE, Bowman S, Paulsen IT, James K, Eisen JA, Rutherford K, Salzberg SL, Craig A, Kyes S, Chan MS, Nene V, Shallom SJ, Suh B, Peterson J, Angiuoli S, Pertea M, Allen J, Selengut J, Haft D, Mather MW, Vaidya AB, Martin DM, Fairlamb AH, Fraunholz MJ, Roos DS, Ralph SA, McFadden GI, Cummings LM, Subramanian GM, Mungall C, Venter JC, Carucci DJ, Hoffman SL, Newbold C, Davis RW, Fraser CM, and Barrell B. 2002. Genome sequence of the human malaria parasite Plasmodium falciparum. Nature 419:498-511. 10.1038/nature01097

Global Malaria Programme WG. 2019. World malaria report 2019.

Guy AJ, Irani V, MacRaild CA, Anders RF, Norton RS, Beeson JG, Richards JS, and Ramsland PA. 2015. Insights into the Immunological Properties of Intrinsically Disordered Malaria Proteins Using Proteome Scale Predictions. PLoS One 10:e0141729. 10.1371/journal.pone.0141729

Haerty W, and Golding GB. 2011. Increased polymorphism near low-complexity sequences across the genomes of Plasmodium falciparum isolates. Genome Biol Evol 3:539-550. 10.1093/gbe/evr045

Harbi D, and Harrison PM. 2014a. Classifying prion and prion-like phenomena. Prion 8:161165. 10.4161/pri.27960

Harbi D, and Harrison PM. 2014b. Classifying prion and prion-like phenomena. Prion 8.

Harbi D, and Harrison PM. 2014c. Interaction networks of prion, prionogenic and prion-like proteins in budding yeast, and their role in gene regulation. PLoS One 9:e100615. 10.1371/journal.pone.0100615

Harbi D, Parthiban M, Gendoo DM, Ehsani S, Kumar M, Schmitt-Ulms G, Sowdhamini R, and Harrison PM. 2012. PrionHome: a database of prions and other sequences relevant to prion phenomena. PLoS One 7:e31785.

Peer] reviewing PDF | (2020:04:48418:1:0:NEW 29 Jul 2020) 
442 Harrison PM. 2017. fLPS: Fast discovery of compositional biases for the protein universe. $B M C$

443

444

445

446

447

448

449

450

451

452

453

454

455

456

457

458

459

460

461

462

463

464

465

466

467

468

469

470

471

472

473

474

475

476

477

478

479

480

481

482

483

484

485

486

487

Bioinformatics 18:476. 10.1186/s12859-017-1906-3

Harrison PM. 2019. Evolutionary behaviour of bacterial prion-like proteins. PLoS One 14:e0213030. 10.1371/journal.pone.0213030

Huntley RP, Harris MA, Alam-Faruque Y, Blake JA, Carbon S, Dietze H, Dimmer EC, Foulger RE, Hill DP, Khodiyar VK, Lock A, Lomax J, Lovering RC, Mutowo-Meullenet P, Sawford T, Van Auken K, Wood V, and Mungall CJ. 2014. A method for increasing expressivity of Gene Ontology annotations using a compositional approach. BMC Bioinformatics 15:155. 10.1186/1471-2105-15-155

Huntley RP, Sawford T, Mutowo-Meullenet P, Shypitsyna A, Bonilla C, Martin MJ, and O'Donovan C. 2015. The GOA database: gene Ontology annotation updates for 2015. Nucleic Acids Res 43:D1057-1063. 10.1093/nar/gku1113

Jiang Y, Oron TR, Clark WT, Bankapur AR, D'Andrea D, Lepore R, Funk CS, Kahanda I, Verspoor KM, Ben-Hur A, Koo da CE, Penfold-Brown D, Shasha D, Youngs N, Bonneau R, Lin A, Sahraeian SM, Martelli PL, Profiti G, Casadio R, Cao R, Zhong Z, Cheng J, Altenhoff A, Skunca N, Dessimoz C, Dogan T, Hakala K, Kaewphan S, Mehryary F, Salakoski T, Ginter F, Fang H, Smithers B, Oates M, Gough J, Toronen P, Koskinen P, Holm L, Chen CT, Hsu WL, Bryson K, Cozzetto D, Minneci F, Jones DT, Chapman S, Bkc D, Khan IK, Kihara D, Ofer D, Rappoport N, Stern A, Cibrian-Uhalte E, Denny P, Foulger RE, Hieta R, Legge D, Lovering RC, Magrane M, Melidoni AN, Mutowo-Meullenet P, Pichler K, Shypitsyna A, Li B, Zakeri P, ElShal S, Tranchevent LC, Das S, Dawson NL, Lee D, Lees JG, Sillitoe I, Bhat P, Nepusz T, Romero AE, Sasidharan R, Yang H, Paccanaro A, Gillis J, Sedeno-Cortes AE, Pavlidis P, Feng S, Cejuela JM, Goldberg T, Hamp T, Richter L, Salamov A, Gabaldon T, Marcet-Houben M, Supek F, Gong Q, Ning W, Zhou Y, Tian W, Falda M, Fontana P, Lavezzo E, Toppo S, Ferrari C, Giollo M, Piovesan D, Tosatto SC, Del Pozo A, Fernandez JM, Maietta P, Valencia A, Tress ML, Benso A, Di Carlo S, Politano G, Savino A, Rehman HU, Re M, Mesiti M, Valentini G, Bargsten JW, van Dijk AD, Gemovic B, Glisic S, Perovic V, Veljkovic V, Veljkovic N, Almeida ESDC, Vencio RZ, Sharan M, Vogel J, Kansakar L, Zhang S, Vucetic S, Wang Z, Sternberg MJ, Wass MN, Huntley RP, Martin MJ, O'Donovan C, Robinson PN, Moreau Y, Tramontano A, Babbitt PC, Brenner SE, Linial M, Orengo CA, Rost B, Greene CS, Mooney SD, Friedberg I, and Radivojac P. 2016. An expanded evaluation of protein function prediction methods shows an improvement in accuracy. Genome Biol 17:184. 10.1186/s13059-016-1037-6

Jones DT, and Cozzetto D. 2015. DISOPRED3: precise disordered region predictions with annotated protein-binding activity. Bioinformatics 31:857-863. 10.1093/bioinformatics/btu744

Le NQK, Yapp EKY, Nagasundaram N, Chua MCH, and Yeh HY. 2019a. Computational identification of vesicular transport proteins from sequences using deep gated recurrent units architecture. Comput Struct Biotechnol J 17:1245-1254. 10.1016/j.csbj.2019.09.005

Le NQK, Yapp EKY, and Yeh HY. 2019b. ET-GRU: using multi-layer gated recurrent units to identify electron transport proteins. BMC Bioinformatics 20:377. 10.1186/s12859-0192972-5

Light S, Sagit R, Sachenkova O, Ekman D, and Elofsson A. 2013. Protein expansion is primarily due to indels in intrinsically disordered regions. Mol Biol Evol 30:2645-2653. $10.1093 / \mathrm{molbev} / \mathrm{mst} 157$

Peer) reviewing PDF | (2020:04:48418:1:0:NEW 29 Jul 2020) 
488

489

490

491

492

493

494

495

496

497

498

499

500

501

502

503

504

505

506

507

508

509

510

511

512

513

514

515

516

517

518

519

520

521

522

523

524

525

526

527

528

529

530

531

532
Lobanov MY, Klus P, Sokolovsky IV, Tartaglia GG, and Galzitskaya OV. 2016. Non-random distribution of homo-repeats: links with biological functions and human diseases. Sci Rep 6:26941. 10.1038/srep26941

Mohan A, Sullivan WJ, Jr., Radivojac P, Dunker AK, and Uversky VN. 2008. Intrinsic disorder in pathogenic and non-pathogenic microbes: discovering and analyzing the unfoldomes of early-branching eukaryotes. Mol Biosyst 4:328-340. 10.1039/b719168e

Muralidharan V, and Goldberg DE. 2013. Asparagine repeats in Plasmodium falciparum proteins: good for nothing? PLoS Pathog 9:e1003488. 10.1371/journal.ppat.1003488

Mutowo-Meullenet P, Huntley RP, Dimmer EC, Alam-Faruque Y, Sawford T, Jesus Martin M, O'Donovan C, and Apweiler R. 2013. Use of Gene Ontology Annotation to understand the peroxisome proteome in humans. Database (Oxford) 2013:bas062. 10.1093/database/bas062

Narasumani M, and Harrison PM. 2015. Bioinformatical parsing of folding-on-binding proteins reveals their compositional and evolutionary sequence design. Sci Rep 5:18586. 10.1038/srep 18586

Nielsen JT, and Mulder FAA. 2019. Quality and bias of protein disorder predictors. Sci Rep 9:5137. 10.1038/s41598-019-41644-w

O'Brien EP, Vendruscolo M, and Dobson CM. 2014. Kinetic modelling indicates that fasttranslating codons can coordinate cotranslational protein folding by avoiding misfolded intermediates. Nat Commun 5:2988. 10.1038/ncomms3988

Pallares I, de Groot NS, Iglesias V, Sant'Anna R, Biosca A, Fernandez-Busquets X, and Ventura S. 2018. Discovering Putative Prion-Like Proteins in Plasmodium falciparum: A Computational and Experimental Analysis. Front Microbiol 9:1737. 10.3389/fmicb.2018.01737

Pancsa R, and Tompa P. 2016. Coding Regions of Intrinsic Disorder Accommodate Parallel Functions. Trends Biochem Sci 41:898-906. 10.1016/j.tibs.2016.08.009

Pizzi E, and Frontali C. 2001. Low-complexity regions in Plasmodium falciparum proteins. Genome Res 11:218-229. 10.1101/gr.gr-1522r

Romero P, Obradovic Z, Li X, Garner EC, Brown CJ, and Dunker AK. 2001. Sequence complexity of disordered protein. Proteins 42:38-48.

Russell K, Cheng CH, Bizzaro JW, Ponts N, Emes RD, Le Roch K, Marx KA, and Horrocks P. 2014. Homopolymer tract organization in the human malarial parasite Plasmodium falciparum and related Apicomplexan parasites. BMC Genomics 15:848. 10.1186/14712164-15-848

Singh GP, Chandra BR, Bhattacharya A, Akhouri RR, Singh SK, and Sharma A. 2004. Hyperexpansion of asparagines correlates with an abundance of proteins with prion-like domains in Plasmodium falciparum. Mol Biochem Parasitol 137:307-319. 10.1016/j.molbiopara.2004.05.016

Su TY, and Harrison PM. 2019. Conservation of Prion-Like Composition and Sequence in PrionFormers and Prion-Like Proteins of Saccharomyces cerevisiae. Front Mol Biosci 6:54. 10.3389/fmolb.2019.00054

Tetz G, and Tetz V. 2017. Prion-Like Domains in Phagobiota. Front Microbiol 8:2239. 10.3389/fmicb.2017.02239

Tetz G, and Tetz V. 2018. Prion-like Domains in Eukaryotic Viruses. Sci Rep 8:8931. 10.1038/s41598-018-27256-w

Peer) reviewing PDF | (2020:04:48418:1:0:NEW 29 Jul 2020) 
533 Uthayakumar M, Benazir B, Patra S, Vaishnavi MK, Gurusaran M, Sureka K, Jeyakanthan J, and

534

535

536

537

538

539

540

541

542

543

544

545

546

547

548

549

550
Sekar K. 2012. Homepeptide repeats: implications for protein structure, function and evolution. Genomics Proteomics Bioinformatics 10:217-225. 10.1016/j.gpb.2012.04.001

Wang E, Wang J, Chen C, and Xiao Y. 2015. Computational evidence that fast translation speed can increase the probability of cotranslational protein folding. Sci Rep 5:15316.

Ward JJ, Sodhi JS, McGuffin LJ, Buxton BF, and Jones DT. 2004. Prediction and functional analysis of native disorder in proteins from the three kingdoms of life. J Mol Biol 337:635-645. 10.1016/j.jmb.2004.02.002

Waudby CA, Dobson CM, and Christodoulou J. 2019. Nature and Regulation of Protein Folding on the Ribosome. Trends Biochem Sci. 10.1016/j.tibs.2019.06.008

Xue HY, and Forsdyke DR. 2003. Low-complexity segments in Plasmodium falciparum proteins are primarily nucleic acid level adaptations. Mol Biochem Parasitol 128:21-32.

Zhou T, Weems M, and Wilke CO. 2009. Translationally optimal codons associate with structurally sensitive sites in proteins. Mol Biol Evol 26:1571-1580. 10.1093/molbev/msp070

Zilversmit MM, Volkman SK, DePristo MA, Wirth DF, Awadalla P, and Hartl DL. 2010. Lowcomplexity regions in Plasmodium falciparum: missing links in the evolution of an extreme genome. Mol Biol Evol 27:2198-2209. 10.1093/molbev/msq108

551 


\section{Table $\mathbf{1}$ (on next page)}

Homopeptides enriched/depleted amino-acid types in protein domains sorted by $p$ values 


\section{Table 1: Homopeptide enriched/depleted amino-acid types in protein domains}

\begin{tabular}{|c|c|c|c|c|c|c|c|c|c|c|c|}
\hline $\begin{array}{l}\text { Amino } \\
\text { Acid } \\
\text { (one } \\
\text { letter } \\
\text { code) }\end{array}$ & $\begin{array}{c}\text { Homop } \\
\text { eptide } \\
\text { amount } \\
(\geq 3) \text { in } \\
\text { pfam } \\
\text { domain } \\
(n=190 \\
52)\end{array}$ & $\begin{array}{c}\text { Fracti } \\
\text { on in } \\
\text { domai } \\
n s\end{array}$ & $\underset{* * * *}{\boldsymbol{P}_{\text {dom }}}$ & $\begin{array}{c}\text { Homop } \\
\text { eptide } \\
\text { amount } \\
(\geq 3) \text { in } \\
\text { proteo } \\
\text { me } \\
(N=209 \\
236)\end{array}$ & $P$-value * & $\begin{array}{c}\text { Homopepti } \\
\text { de amount } \\
(\geq 5) \text { in } \\
\text { pfam } \\
\text { domain } \\
(n=2121)\end{array}$ & $\begin{array}{c}\text { Homop } \\
\text { eptide } \\
\text { amount } \\
(\geq 5) \text { in } \\
\text { proteo } \\
\text { me } \\
(N=613 \\
33)\end{array}$ & $P$-value & $\begin{array}{l}\text { Homop } \\
\text { eptide } \\
\text { amount } \\
(\geq 10) \text { in } \\
\text { pfam } \\
\text { domain } \\
(n=349)\end{array}$ & $\begin{array}{c}\text { Homop } \\
\text { eptide } \\
\text { amount } \\
(\geq 10) \text { in } \\
\text { proteo } \\
\text { me } \\
(N=177 \\
24)\end{array}$ & $P$-value \\
\hline $\mathbf{A}$ & 723 & 0.76 & +0.92 & 954 & 0 & 0 & 0 & 1 & 0 & 0 & 1 \\
\hline $\mathbf{L}$ & 1676 & 0.20 & +0.34 & 8250 & $2.44 e-222$ & $\underline{0}$ & $\underline{261}$ & $\underline{0.00010}$ & 0 & 0 & 1 \\
\hline I & 1527 & 0.21 & +0.36 & 7315 & $4.69 e-214$ & 0 & 0 & 1 & 0 & 0 & 1 \\
\hline $\mathbf{G}$ & 579 & 0.38 & +0.62 & 1527 & $1.30 \mathrm{e}-206$ & 0 & 125 & 0.01223 & 0 & 0 & 1 \\
\hline $\mathbf{V}$ & 378 & 0.34 & +0.57 & 1108 & $4.13 e-118$ & 0 & 0 & 1 & 0 & 0 & 1 \\
\hline $\mathbf{R}$ & 454 & 0.28 & +0.49 & 1606 & $1.72 \mathrm{e}-108$ & 0 & 121 & 0.01408 & 0 & 0 & 1 \\
\hline$T$ & 417 & 0.18 & +0.30 & 2341 & $2.67 e-40$ & 15 & 318 & 0.05316 & 0 & 0 & 1 \\
\hline $\mathrm{K}$ & 5036 & 0.10 & +0.04 & 48223 & $1.81 \mathrm{e}-31$ & 509 & 10312 & $1.95 e-18$ & 0 & 0 & 1 \\
\hline S & 1059 & 0.11 & +0.08 & 9511 & $9.57 e-13$ & 75 & 1167 & $1.51 \mathrm{e}-07$ & 0 & 146 & 0.05417 \\
\hline $\mathbf{P}$ & 131 & 0.17 & +0.27 & 774 & $2.45 e-12$ & 0 & 129 & 0.01062 & 0 & 0 & 1 \\
\hline$F$ & 481 & 0.12 & +0.12 & 3913 & $4.00 e-12$ & 0 & 144 & 0.00626 & 0 & 0 & 1 \\
\hline $\mathbf{Y}$ & 393 & 0.11 & +0.08 & 3582 & $1.54 \mathrm{e}-05$ & $\underline{0}$ & $\underline{190}$ & $\underline{0.00123}$ & 0 & 0 & 1 \\
\hline C & 27 & 0.18 & +0.30 & 148 & 0.00022 & 0 & 5 & 0.83864 & 0 & 0 & 1 \\
\hline$Q$ & 121 & 0.11 & +0.08 & 1098 & 0.00384 & 15 & 256 & 0.01668 & $\underline{0}$ & 107 & 0.11832 \\
\hline
\end{tabular}




\begin{tabular}{|c|c|c|c|c|c|c|c|c|c|c|c|}
\hline $\mathrm{E}$ & 1170 & 0.09 & -0.01 & 12572 & 0.00914 & 65 & 1907 & 0.05070 & $\underline{\mathbf{0}}$ & $\underline{\mathbf{3 8 0}}$ & $\underline{\mathbf{0 . 0 0 0 4 8}}$ \\
\hline $\mathrm{W}$ & 3 & 0.33 & +0.56 & 9 & 0.03576 & 0 & 0 & 1 & 0 & 0 & 1 \\
\hline $\mathrm{M}$ & 22 & 0.12 & +0.12 & 189 & 0.04567 & 0 & 0 & 1 & 0 & 0 & 1 \\
\hline$\underline{\mathbf{N}}$ ** & $\underline{\mathbf{3 9 4 1}}$ & $\underline{\mathbf{0 . 0 4}}$ & $\underline{\mathbf{- 0 . 3 6}}$ & $\underline{\mathbf{9 2 7 2 2}}$ & $\underline{\mathbf{0}}$ & $\underline{\mathbf{1 3 6 3}}$ & $\underline{\mathbf{4 3 6 3 2}}$ & $\underline{\mathbf{5 . 1 1 e - 1 3}}$ & $\mathbf{3 4 9}$ & $\mathbf{1 6 6 2 7}$ & $\mathbf{1 . 6 5 e - 1 0}$ \\
\hline$\underline{\mathbf{D}}$ & $\underline{\mathbf{8 8 1}}$ & $\underline{\mathbf{0 . 0 7}}$ & $\underline{\mathbf{0 . 1 1}}$ & $\underline{\mathbf{1 2 6 3 6}}$ & $\underline{\mathbf{7 . 8 3 e - 2 0}}$ & 79 & 2642 & 0.01802 & $\underline{\mathbf{0}}$ & $\underline{\mathbf{4 6 4}}$ & $\underline{\mathbf{8 . 6 8 e - 0 5}}$ \\
\hline$\underline{\mathbf{H}}$ & $\underline{\mathbf{3 3}}$ & $\underline{\mathbf{0 . 0 4}}$ & $\underline{\mathbf{0 . 3 6}}$ & $\underline{\mathbf{7 5 8}}$ & $\underline{\mathbf{2 . 3 1 e - 0 7}}$ & 0 & 124 & 0.01267 & 0 & 0 & 1 \\
\hline
\end{tabular}

2

3

4 * P-value threshold $=\mathbf{0 . 0 0 2 5}$ (with a Bonferroni correction accounting for tests on the twenty amino acids). $\mathbf{P}$ -

5 values of 0.0 are infinitesimally small beyond the precision of the computation.

6 ** Significant enrichments or depletions are in bold. Underlined ones are homopeptide-depleted amino acids.

$7^{* * *} \mathbf{P}_{\text {dom }}$ is the propensity of homopeptides of a specific amino-acid type to occur in protein domains. It is

8 calculated as described in Methods. 
Table 2 (on next page)

Enrichment of homopeptides within protein domains that are near or overlapping IDRs 
1 Table 2: Enrichment of homopeptides within protein domains that

2 are near or overlapping IDRs

3

4

\begin{tabular}{|c|c|c|c|c|c|c|c|}
\hline $\begin{array}{l}\text { Intrinsic } \\
\text { disorder } \\
\text { annotator }\end{array}$ & $\begin{array}{l}\text { Total } \\
\text { number } \\
\text { of } \\
\text { domain } \\
\text { residues } \\
\text { in/near } \\
\text { IDRs }\end{array}$ & $\begin{array}{l}\text { Homopeptide } \\
\text { residues } \\
\text { in/near IDRs }\end{array}$ & $\begin{array}{l}\text { Total } \\
\text { number } \\
\text { of } \\
\text { domain } \\
\text { residues }\end{array}$ & $\begin{array}{c}\text { Total number } \\
\text { of } \\
\text { homopeptides } \\
\text { in domains }\end{array}$ & $\begin{array}{c}\text { P- } \\
\text { value* }\end{array}$ & ** & 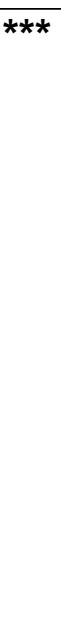 \\
\hline IUPred2A & 38940 & 2845 & 808565 & 19052 & 0.0 & 49 & 1 \\
\hline DisoPred3 & 15928 & 1381 & 808565 & 19052 & 0.0 & 29 & 1 \\
\hline
\end{tabular}

5 * These P-value results are not affected by chopping off 3, 5 or 7 residues from the

6 ends of the protein domains, as for Table S3.

$7{ }^{* *}$ Total number of individual protein domains that have enrichment of homopeptides

8 within their parts that are near or overlapping IDRs.

$9{ }^{* * *}$ Total number of individual protein domains that have depletion of homopeptides

10 within their parts that are near or overlapping IDRs 


\section{Figure 1}

Schematic of the analysis.

Homopeptides were defined as $\geq 3$ consecutive amino acids of the same type in a sequence. Protein domains near intrinsically disordered regions (IDRs) were determined using a 10residues buffer. Also, if the IDR is within a protein domain or otherwise overlaps it, a 10residue buffer is considered on either side of the IDR as shown. 


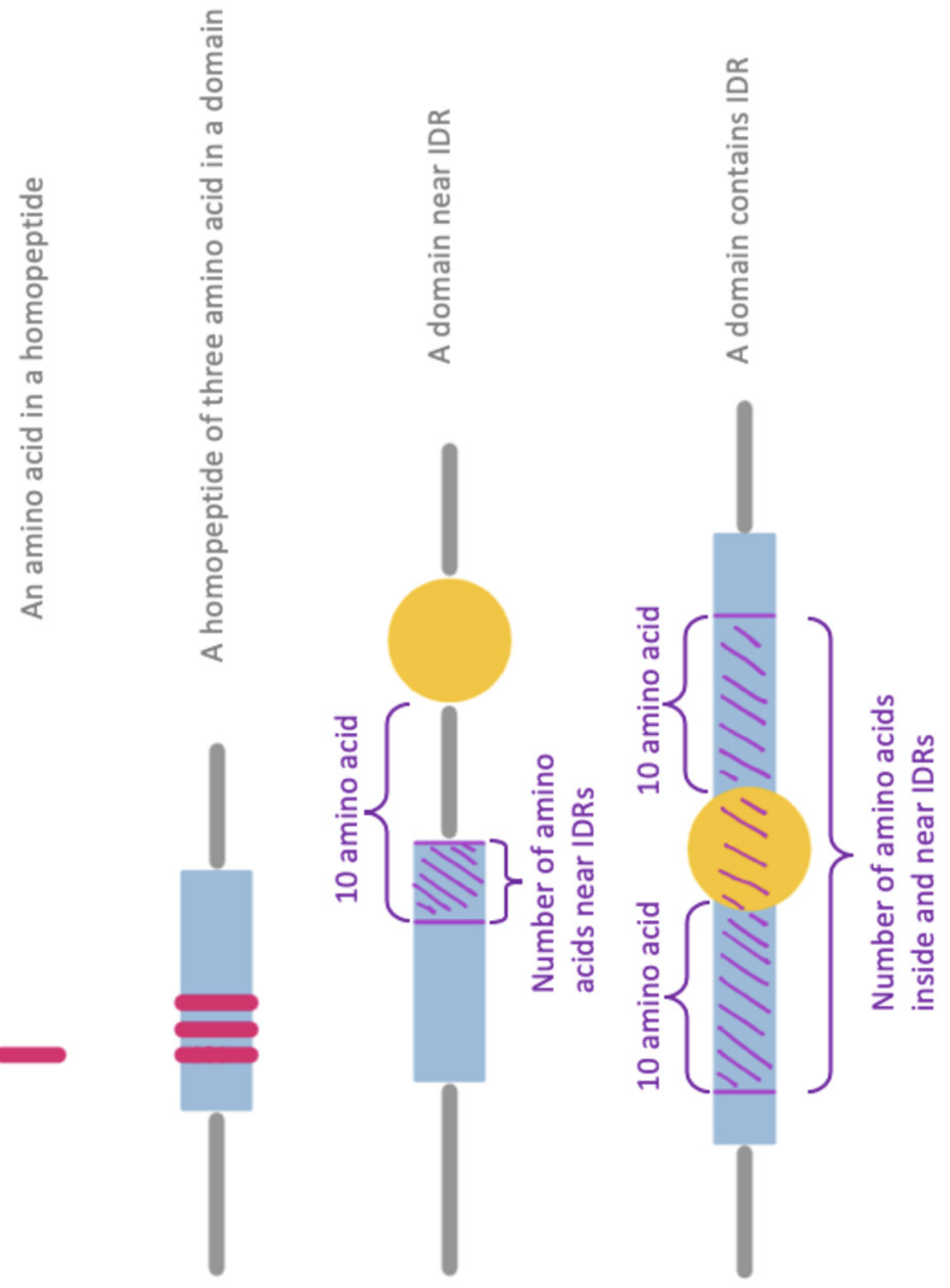


Figure 2

Distribution of homopeptide length inside and outside of protein domains.

(a) The distribution of homopeptide length for all residues both inside and outside protein domains. The natural log of the total number of homopeptides for a given length is used. Parts (b), (c), (d) are the same distributions but for K-, N-, and Dhomopeptides respectively. 
a.

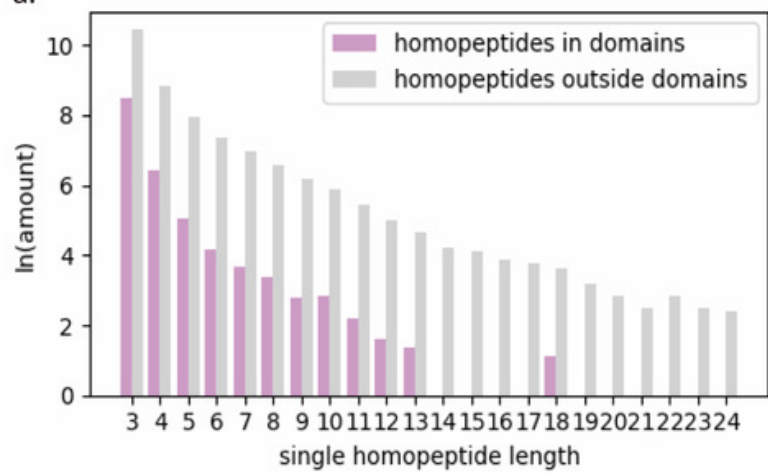

c.

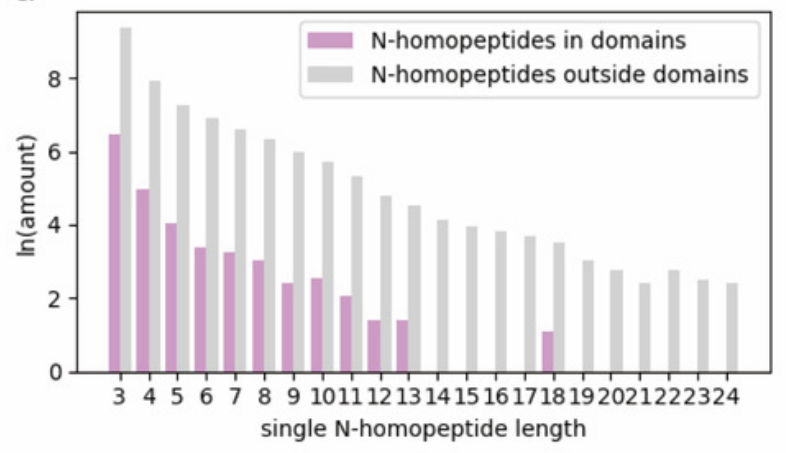

b.

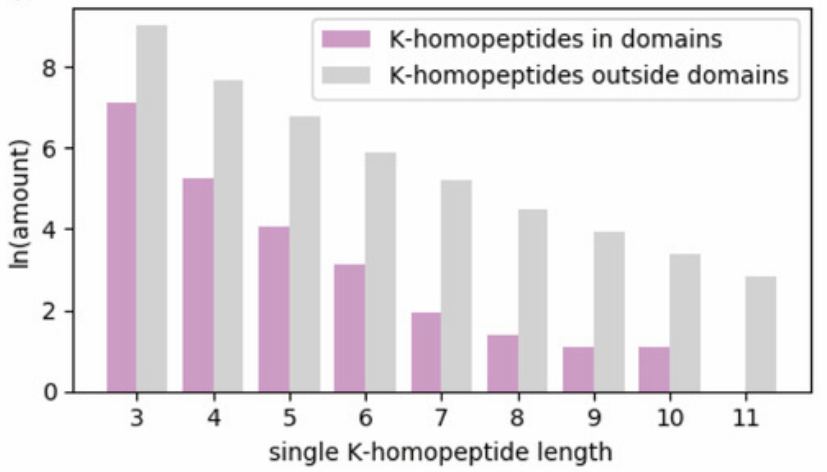

d.

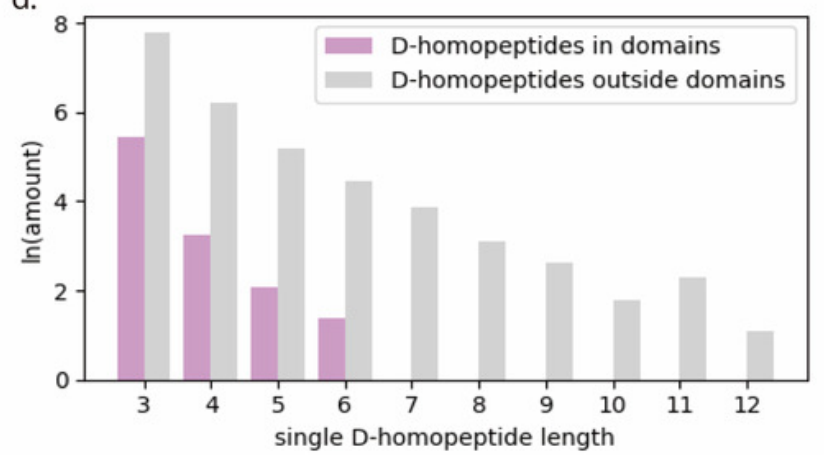

